\title{
UK Renal Registry 13th Annual Report (December 2010): Appendix C Renal Services Described for Non-Physicians
}

This appendix provides information on the issues discussed in this report, background information on renal failure and discusses the services available for its treatment.

\section{The role of the kidneys}

1.1 The kidneys are paired organs located behind the abdominal cavity, in the retroperitoneal space. Their primary function is to produce urine, which allows the removal of metabolism-related waste products from the blood. The kidneys also have a role in controlling fluid balance and blood pressure, red blood cell production and the maintenance of healthy bones.

\section{Kidney diseases}

1.2 Around 13,000 people die from kidney (renal) disease in the UK each year, though this is an underestimation as many deaths of patients with renal failure are not recorded as such in mortality statistics. Kidney diseases can occur suddenly ('acute') or over months and years ('chronic'). Chronic kidney disease is relatively common, with the majority of patients being elderly and having mild impairment of their renal function.

\section{Acute kidney injury}

1.3 Acute kidney injury (AKI) has replaced the previous term 'acute renal failure'. AKI, which is often a reversible process, occurs when there is a rapid loss of renal function due to kidney damage. The causes of AKI can be divided into 3 categories: pre-renal (interference with the renal blood supply), intrinsic (damage to the kidney itself) and post-renal (obstructive causes in the urinary tract). Some patients with AKI require dialysis for a few days or weeks until their renal function improves, though a small proportion of individuals never recover kidney function. AKI normally occurs in the context of other illness and patients are often unwell; approximately 50\% of patients with AKI who receive dialysis do not survive.

\section{Chronic kidney disease (CKD) and established renal failure (ERF)}

1.4 Chronic kidney disease affects approximately 3 million people in the UK and occurs because of slow damage to the kidneys over a number of months or years. The incidence increases with age and is higher in certain ethnic groups, such as patients of South Asian and African descent. In the initial stages of CKD, patients are usually well and there is little to find on clinical examination. Early abnormal findings may include blood (haematuria) and protein (proteinuria) in the urine or elevated blood pressure (hypertension). However, the lack of symptoms means many patients present to medical services with advanced disease. In the latter stages of CKD, patients may complain of tiredness, a loss of appetite, feeling

\section{KARGER \\ Fax +4161306 1234 E-Mail karger@karger.ch} www.karger.com
UK Renal Registry, Southmead Hospital, Southmead Road, Bristol, BS10 5NB, UK Email: clare@renalreg.com 
sick (nausea) and itching (pruritus). Other symptoms, such as ankle swelling (oedema), may be present depending on the underlying condition causing CKD.

1.5 Other terms used for chronic kidney disease include chronic renal impairment, chronic renal insufficiency and chronic renal failure. Established renal failure (ERF) refers to kidney function that has deteriorated to a level where treatment is required to sustain life. Treatment options include dialysis and renal transplantation but some patients decide not to receive dialysis and opt for conservative management. This involves input from specialist nurses and palliative care services, and focuses on treating the complications of kidney disease and managing symptoms.

\section{Causes of CKD}

1.6 Most renal diseases that cause renal failure fall into five categories.

1. Generalised (systemic) disease. Diabetes mellitus is by far the most common systemic disease that affects the kidneys (around 20\% of all renal disease). Diabetic patients often develop progressive kidney damage over many years, particularly if blood glucose levels and blood pressure are poorly controlled. Careful lifelong supervision of diabetes has a major impact in preventing kidney damage. Other systemic diseases that can cause kidney damage include autoimmune conditions (e.g. systemic lupus erythematous and vasculitis), amyloidosis and multiple myeloma.

2. Glomerulonephritis. This term describes conditions that damage the glomeruli (the filtering units of the kidneys that start the process of urine formation). There are many different causes of glomerulonephritis and treatment depends on the form of the disease. Some types of glomerulonephritis are relatively benign and unlikely to progress to established renal failure. Other forms are more aggressive with treatment making only a small impact on disease progression and the development of established renal failure.
3. High blood pressure (hypertension). Severe ('accelerated') hypertension causes chronic kidney disease, but early recognition and treatment of high blood pressure can halt - and to some extent reverse - the associated kidney damage. Hypertension is a common cause of renal failure in patients of African origin.

4. Obstruction. CKD can be a consequence of any pathology that obstructs the free flow of urine through the urinary system. Most often obstruction is secondary to enlargement of the prostate gland in elderly men, but other causes include kidney stones, bladder tumours, and congenital abnormalities of the renal tract.

5. Genetic disease. The commonest genetic disease causing CKD is polycystic kidney disease. This condition, along with many rare inherited diseases affecting the kidneys, accounts for about $8 \%$ of all kidney failure in the UK.

\section{Prevention and management}

1.8 Within the UK, risk factors for $\mathrm{CKD}$, such as diabetes, obesity and hypertension are becoming more common. Consequently, the NHS is increasingly focusing on the prevention and early detection and treatment of CKD. Although many of the diseases causing CKD are not preventable, their recognition is important to allow appropriate treatment of any complications and preparation for renal replacement therapy. Some diseases, such as urinary obstruction, may be reversible to some extent and intervention is appropriate. Good diabetic control and blood pressure management may halt the rate of future renal function decline.

1.9 Clear guidelines are in place for the management of CKD by both general practitioners and hospital kidney specialists (nephrologists) [1]. Currently there is no general population screening for renal disease; instead, targeted screening of patients groups 'at-risk' of renal disease, such as diabetic or hypertensive patients, occurs. This normally involves testing the urine for the presence of blood or protein, plus blood tests for the level of substances normally excreted by the kidney such as creatinine and urea. 


\section{Complications and comorbidity}

1.10 Patients with chronic kidney disease often have accompanying illnesses (comorbidities). Some are due to the primary disease, e.g. diabetes may cause blindness and diseases of the nerves and blood vessels. Others, such as anaemia, bone disease, and heart failure, are consequences of the renal failure. In addition, many patients with established renal failure, have diseases affecting the heart and blood vessels (vascular) particularly ischaemic heart disease and peripheral vascular disease. Comorbidity, can influence the choice of treatment for renal failure and may reduce its benefits. Early and aggressive management of CKD-related complications, such as bone mineral abnormalities (hyperparathyroidism), may reduce the incidence of vascular disease.

\section{Renal replacement therapy}

1.11 The term renal replacement therapy (RRT) encompasses the three treatments used in established renal failure: haemodialysis, peritoneal dialysis and kidney transplantation. Both forms of dialysis remove waste products from the blood, but the other complications of established renal failure, such as anaemia and abnormal bone metabolism (hyperparathyroidism), require treatment with medications. Patients under the age of around 70 may undergo kidney transplantation as a form of treatment. If successful, a kidney transplant returns an individual to good health and removes the need for dialysis.

\section{Renal dialysis}

1.12 Dialysis involves the removal of waste products from the blood by allowing these products to diffuse across a thin membrane into dialysis fluid, which is then discarded along with the toxic waste products. The fluid is chemically composed to draw or 'attract' excess salts and water from the blood to cross the membrane, without the blood itself being in contact with the fluid.

\section{Haemodialysis}

1.13 The method first used to achieve dialysis was the artificial kidney, or haemodialysis. This involves the attachment of the patient's circulation to a machine through which fluid is passed and exchange can take place. A disadvantage of this method is that some form of permanent access to the circulation must be produced to be used at every treatment. The majority of patients on haemodialysis receive three four-hour sessions a week, at either a hospital-based dialysis unit or a community-based unit (satellite unit) away from the main renal centre. A small number of patients perform their own dialysis at home (home haemodialysis) and the number and duration of treatments will vary.

\section{Peritoneal dialysis}

1.14 An alternative form of dialysis is peritoneal dialysis, most commonly in the form of continuous ambulatory peritoneal dialysis (CAPD). In this technique, dialysis fluid is inserted, via a plastic tube (catheter), into the peritoneal cavity (which lies around the bowel) for approximately 6 hours before being removed and replaced. The fluid must be sterile in order to avoid infection and inflammation of the peritoneum (peritonitis), which is the main complication of the treatment. Each fluid exchange takes 30 to 40 minutes to perform and is repeated three or four times daily.

\section{Renal transplantation}

1.15 Renal transplantation replaces all the kidneys' functions, so erythropoietin and vitamin D supplementation are unnecessary. Transplantation involves the placement of a single kidney in the pelvis, close to the bladder, to which the ureter is connected. The immediate problem is the body's immune system recognising the new organ as foreign tissue-a process known as rejection. Consequently, all patients receiving a kidney transplant require anti-rejection drugs, such as tacrolimus, cyclosporin, and Mycophenolate Mofetil, for the lifetime of the 
transplant. These drugs, known as immunosuppressants, have many undesirable side effects, including the acceleration of vascular disease, increased risk of infection and higher rates of cancer (malignancy). This often means that myocardial infarctions and strokes are commoner in transplant patients than in healthy individuals of the same age. As transplants get older, there is a progressive loss of function due to chronic rejection (chronic allograft nephropathy). The average lifespan of a kidney transplant is between 10 and 15 years, which means some younger patients, will receive more than one transplant during their lifetime, often with periods of dialysis in-between.

1.16 For many patients, renal transplantation, from both live and deceased donors, is the best treatment in terms of survival and quality of life. Unfortunately, despite changes in policy and legislation there remains a shortage of kidneys for transplant; it appears likely that whatever social and medical structures are present, there will inevitably be a shortage of kidneys from humans.

\section{Nature of renal services}

1.17 The work of a nephrologist includes the early detection and diagnosis of renal disease and the longterm management of its complications such as high blood pressure, anaemia and bone disease. The nephrologist may share the management with the general practitioner or local hospital physician; relying on them to refer patients early for initial diagnosis and specific treatment. At any one time, perhaps only $5 \%$ of patients under their care are inpatients in wards with a further $20 \%$ attending the renal centre regularly for haemodialysis. However, inpatient nephrology and the care of patients receiving centre-based dialysis are specialised, complex and require experienced medical advice to be available on a 24 hour basis. Other renal work is sustained on an outpatient basis; this includes renal replacement therapy by dialysis and the care of transplant patients.

1.18 There are six major components to renal medicine.
1. Renal replacement therapy. The most significant element of work relates to the preparation of patients with advanced CKD for RRT and their medical supervision for the remainder of their lives. The patient population will present increasing challenges for renal staffing as more elderly and diabetic patients are accepted for treatment.

2. Emergency work. The emergency work associated with the specialty consists of:

i. Treatment of acute renal failure, often involving multiple organ failure and acute-onchronic renal failure. Close co-operation with other medical specialties, including critical care, is therefore a vital component of this aspect of the service.

ii. Management of medical emergencies arising from an established renal failure programme. This workload is expanding as the number, age, and comorbidity of patients starting renal replacement therapy increases.

3. Routine nephrology. A substantial workload is associated with the immunological and metabolic nature of renal disease which requires investigative procedures in an inpatient setting. It is estimated that ten inpatient beds per million of the population are required for this work.

4. Investigation and management of fluid and electrolyte disorders. This makes up a variable proportion of the nephrologists work, depending on the other expertise available in the hospital.

5. Outpatient work. The outpatient work in renal medicine consists of the majority of general nephrology together with clinics for dialysis and renal transplant patients.

6. Research activities. Many nephrologists have clinical or laboratory-based research interests.

\section{References}

1 National Collaborating Centre for Chronic Conditions. Chronic kidney disease: national clinical guideline for early identification and management in adults in primary and secondary care. London: Royal College of Physicians, September 2008 